\title{
Indicadores de Avaliação em Gestão e Saúde Coletiva na Formação Médica
}

\author{
Evaluation Indicators in Management and \\ Collective Health in Medical Education
}

\author{
Elza de Fátima Ribeiro Higal \\ Maria Lourdes Marmorato Botta Hafner \\ Silvia Franco da Rocha Tonhom \\ Elane de Fátima Taipeiro ${ }^{I}$ \\ Haydée Maria Moreiral \\ Ana Paula Ceolotto Guimarães \\ Osni Lázaro Pinheiro
}

PALAVRAS-CHAVE:

- Indicadores;

- Avaliação Educacional;

- Avaliação de Desempenho.

\section{KEYWORDS:}

- Indicators;

- Educational Measurement;

- Employee Performance Appraisal.
Recebido em:10/10/2012

Aprovado em:30/10/2013

REVISTA BRASILEIRA DE EDUCAÇÃO MÉDICA

\section{RESUMO}

A Faculdade de Medicina de Marília (Famema) utiliza metodologias ativas de ensino-aprendizagem, e sua organização curricular é orientada ao desenvolvimento de competência profissional e a um processo avaliativo critério-referenciado. Esta pesquisa tem por objetivo elaborar indicadores para avaliar o processo de ensino-aprendizagem nos âmbitos da gestão e da saúde coletiva, baseados na adaptação da proposta de Conferência de Consenso e descrever o caminho percorrido nessa elaboração. Com base na análise dos documentos institucionais que explicitavam o planejamento das atividades (cadernos de organização das séries) e nos critérios, formatos e instrumentos da avaliação nas diferentes séries e cenários de aprendizagem (Manual de Avaliação), foi elaborada a primeira matriz de indicadores, que continha a proposta curricular para o alcance da aprendizagem que embasara a análise dos especialistas e a realização da Conferência. Tal metodologia possibilitou entender, ampliar e aprofundar a avaliação nessas áreas específicas, o que poderá orientar a implementação das alterações curriculares necessárias, com ênfase em uma avaliação da aprendizagem comprometida com o processo formativo.

Marilia Medical School uses active methodologies of teaching-learning. Its curriculum organization is directed towards professional competence and to a criterion-referenced evaluative process. This research aims at elaborating indicators to evaluate teaching-learning processes in health management and collective health. These indicators are based on the adaptation of the Consensus Conference proposal; it also aims at describing the pathway followed in this elaboration. The first matrix of indicators showed the proposed curriculum as able to support the specialists' analyses and the organization of the Conference. It was based on the analysis of the institutional documents which explained the activities plan (notebooks on grades organization) and the criteria, formats and evaluations tools in different grades and learning scenarios (Evaluation Handbook). This methodology made it possible to understand the evaluation of these specific areas more deeply and widely, which may guide a better implementation of the necessary curriculum alterations which emphasize an evaluation committed with the formative process. 


\section{INTRODUÇÃO}

A formação médica no Brasil caminha no sentido de se adequar às Diretrizes Curriculares Nacionais (DCN), que instituem o perfil do egresso/profissional tendo em vista uma formação generalista, humanista, crítica e reflexiva, voltada para promoção, prevenção, recuperação e reabilitação da saúde. Tal formação está alicerçada em princípios éticos, de responsabilidade social e de compromisso com a cidadania. Contempla, ainda, para o curso de Medicina, os seguintes saberes: atenção à saúde, tomada de decisões, comunicação, liderança, administração e gerenciamento, e educação permanente ${ }^{1}$. Pressupõe, também, a construção do conhecimento integrando as dimensões biológicas, psicológicas e sociais a partir da realidade, o que pode favorecer o desenvolvimento do saber, do fazer e do ser humano, estando tal formação em consonância com as diretrizes do Sistema Único de Saúde (SUS) ${ }^{2}$.

Para Cecim e Pinto ${ }^{3}$, o processo de formação de recursos humanos em saúde deve ter como pilares fundamentais o pensamento crítico e produtivo, o ensino em consonância com o serviço, a conscientização do estudante frente à realidade, o aprendizado baseado nos problemas da população, de modo que o profissional atue como sujeito transformador da realidade. O papel das instituições de nível superior deve ultrapassar os limites, até então considerados oficiais, de meras reprodutoras intramuros de saberes e permitir a composição entre erudição e criatividade, tão necessária à resolução dos problemas de saúde.

Além disso, estudos indicam a necessidade de ressignificar a avaliação para torná-la uma atividade contínua, sistematizada e inerente ao processo educacional. É necessário reconhecê-la como uma das atividades educacionais mais expressivas do ponto de vista da mobilização dos aprendizes, ampliá-la pela análise integradora dos atributos pessoais em relação aos resultados obtidos e, desta forma, orientada à avaliação da prática profissional ${ }^{4}$.

Segundo Lima ${ }^{4}$, a fragilidade do processo de avaliação pode ser revelada pela ocorrência de falha na formação ou pode estar relacionada a fatores intrínsecos referentes aos valores pessoais, que podem ser incompatíveis com uma postura ética e de responsabilidade social no exercício da profissão. Se a avaliação focalizar apenas os aspectos cognitivos (saber) e/ou as habilidades e destrezas (fazer), a inadequação desse processo estará mantida, uma vez que reduz e fragmenta a avaliação de competência. Assim, a avaliação ocupa um espaço estratégico tanto no desenvolvimento e na melhoria do processo ensino-aprendizagem, como na própria gestão, sendo um eixo estruturante nas mudanças curriculares. Finalmente, deve-se assegurar a coerência entre as avaliações de processo (formativas) e as avaliações que definem a progressão dos estudantes (somativas) e de ambas com o perfil profissional desejado e os princípios filosóficos, pedagógicos e ideológicos do programa ${ }^{4}$.

Na Famema, uma ampla pesquisa, realizada com os egressos do curso de Medicina, com os usuários e gestores do serviço de saúde, evidenciou a positividade do redirecionamento curricular que a instituição assumiu. Na percepção dos egressos, o curso proporcionou uma formação humanista, promoveu o aprender a aprender, valorizou a convivência com outros profissionais e integrou a teoria com a prática. As pessoas atendidas pelos egressos os consideraram cuidadosos, respeitosos e capazes de ouvi-las, enquanto os gestores reconheceram que a atuação do egresso ia além do foco da doença. No entanto, na percepção dos egressos, o curso também apresentou limites ${ }^{5}$ que evidenciam a necessidade de construir um processo avaliativo embasado em indicadores que representem a realidade dos cenários em que o estudante está inserido.

Considerando a estrutura curricular da Famema, os resultados encontrados na pesquisa e os indicados nas DCN para avaliação da formação médica, em seu artigo 13, nos incisos 1 e 2

$\S 1^{\circ}$ As avaliações dos alunos deverão basear-se nas competências, habilidades e conteúdos curriculares desenvolvidos, tendo como referência as Diretrizes Curriculares.

$\S 2^{\circ}$ O Curso de Graduação em Medicina deverá utilizar metodologias e critérios para acompanhamento e avaliação do processo ensino-aprendizagem e do próprio curso, em consonância com o sistema de avaliação e a dinâmica curricular definido pela IES à qual pertence (p. 5-6).

é necessário elaborar indicadores de avaliação que, segundo Minayo $^{6}$, atuam como sinalizadores da realidade, uma vez que servem para detalhar se os objetivos de uma proposta estão sendo bem conduzidos, no sentido de avaliação de processo, ou se foram alcançados, visando à avaliação de resultados. Minayo aponta também que a utilidade de bons indicadores depende de sua condição histórica e da regularidade com que são produzidos, de modo que permitam visualizar tendências temporais e que sejam pactuados por grupos e instituições.

Almeida e colaboradores ${ }^{7}$ também reconhecem a necessidade de avanço no processo avaliativo, tendo em vista o 
desenvolvimento de competência profissional e habilidades gerais e específicas requeridas da formação médica, de forma que a avaliação contemple a integralidade e as novas tecnologias do cuidado em saúde.

Nesse contexto, a Famema, que já desenvolve um processo avaliativo critério-referenciado, reconhece a necessidade de aprofundamento dos indicadores de avaliação por ser uma instituição de ensino, pesquisa e assistência, além de a formação de seus profissionais estar em consonância com as diretrizes do SUS.

Assim, a Famema se orienta no sentido de aprimorar a avaliação nos âmbitos da gestão e saúde coletiva para a formação médica. Devido à escassez de estudos sobre o tema, acredita que isso deva ser feito por meio de critérios e indicadores estrategicamente formulados, considerando o cenário de aprendizagem, de modo que contribuam efetivamente para a excelência da formação requerida.

Portanto, a presente pesquisa busca contribuir para ampliar e aprofundar a avaliação do processo ensino-aprendizagem nos diferentes cenários em que são desenvolvidos os saberes relativos à gestão e à saúde coletiva, com base na estruturação de critérios e indicadores de avaliação.

\section{OBJETIVO}

Elaborar indicadores para avaliar o processo de ensino-aprendizagem nas áreas de gestão e saúde coletiva do curso de Medicina da Famema e descrever o caminho percorrido nessa elaboração.

\section{MÉTODO}

Trata-se de uma pesquisa descritiva de cunho qualitativo, que utiliza a técnica da Conferência de Consenso, permitindo que a discussão ocorra com densidade, com a apresentação de evidências disponíveis e sob diferentes pontos de vista ${ }^{8}$. A pesquisa foi aprovada pelo Comitê de Ética em Pesquisa da Famema sob o protocolo 791/10.

Foi realizada a seleção, por meio de uma amostra de conveniência, de um grupo de seis especialistas para se promoverem as discussões em busca de consenso. O grupo selecionado não foi extenso, para que fosse assegurada a participação individual numa discussão ampliada e aprofundada acerca dos indicadores. Esses especialistas são profissionais que possuem expertise em currículos de cursos com metodologias ativas e/ou têm vivência no acompanhamento da prática de estudantes da Famema em diversos cenários, tais como enfermeiros/médicos assistenciais, gestores, preceptores e docentes. $\mathrm{O}$ trabalho foi desenvolvido em quatro fases, descritas a seguir.

\section{Primeira fase}

Foi realizada a análise documental dos manuais de cada série e a dos formatos de avaliação do Exercício de Avaliação da Prática Profissional (EAPP) que constam no Manual de Avaliação do Curso de Medicina da Famema, para elaboração de uma matriz inicial que contemplasse a proposta de ensino-aprendizagem, delineada em cada série para a graduação, no que se refere à área da gestão e à saúde coletiva.

\section{Segunda fase}

A matriz inicial foi enviada, via on-line e correio postal, juntamente com o Termo de Consentimento Livre Esclarecido, para os especialistas. Foi-lhes solicitada a avaliação dos indicadores propostos, com atribuição de pontos de 0 a 10, com 0 correspondendo à discordância completa $\mathrm{e} 10$ equivalendo à máxima concordância, e com possibilidade de apontarem sugestões de alterações e/ou inclusões, com o prazo de 30 dias para devolução.

Após esse prazo, as avaliações individuais dos especialistas foram consolidadas em uma nova matriz e foram incorporadas as sugestões de readequação encaminhadas. As notas atribuídas foram consolidadas no programa Excel (Microsoft Corporation, EUA). Para cada indicador, calculou-se a média aritmética (medida de sua importância) e o desvio padrão (estimativa do grau de consenso entre os avaliadores). Nesse momento, não se excluiu nenhum indicador, a fim de levar para a conferência de consenso todas as informações para discussão.

\section{Terceira fase}

Realizou-se a conferência, com cinco especialistas presentes, na qual se apresentou a consolidação das avaliações para cada indicador. Cada participante pôde expressar seu entendimento sobre o indicador, seus argumentos e dar sugestões. Tal discussão buscou a compreensão da matriz, bem como a dos sentidos a ela subjacentes, para que se estabelecesse, ou não, um consenso para a construção dos indicadores do processo ensino-aprendizagem nas áreas de gestão e de saúde coletiva. Após a obtenção do consentimento dos participantes, a conferência foi gravada e filmada, e as falas posteriormente transcritas para análise dos argumentos que orientaram a reformulação dos indicadores.

Após a análise da conferência, a matriz foi reorganizada conforme as adequações sugeridas e encaminhada novamente aos especialistas para nova rodada de avaliação individual com atribuição de pontos.

\section{Quarta fase}

Foi feito o tratamento estatístico das avaliações dos especialistas no programa Excel por meio de média aritmética e desvio padrão. 
Segundo Souza e colaboradores ${ }^{8}$, estabeleceram-se como critérios de corte: média igual ou superior a sete como indicador importante; desvio padrão inferior a três, considerado consensual; e indicador com média igual ou superior a sete e desvio padrão igual ou superior a três considerado importante, porém não consensual.

Na primeira medida, busca-se estabelecer o grau de importância de cada indicador, enquanto na segunda obtém-se o nível de consenso no conjunto dos indicadores. Por fim, procedeu-se à elaboração dos indicadores, segundo o consenso alcançado na conferência, para avaliar o processo de ensino-aprendizagem do curso de Medicina da Famema nas áreas de gestão e de saúde coletiva.

\section{RESULTADOS E DISCUSSÃO}

$\mathrm{Na}$ primeira fase da pesquisa, em que se analisaram os documentos institucionais para a construção da matriz inicial, as informações buscadas eram referentes ao planejamento da aprendizagem, conhecimentos a serem adquiridos ao longo do curso, ações e tarefas para atingir o desempenho esperado e o alcance da competência profissional. Quanto à avaliação, consultou-se o Manual de Avaliação, que explicita sua conceituação, finalidade e critérios, e descreve os formatos e instrumentos a serem utilizados em todas as séries e nos diferentes cenários.

Essa análise permitiu organizar e estruturar, nesta matriz, a proposta curricular pela qual a Famema orienta o alcance da aprendizagem requerida na formação médica nas áreas da gestão e da saúde coletiva, proposta embasada nos preceitos das DCN. A proposta avaliativa do currículo orientado para o desenvolvimento de competência, em nossa instituição, norteia-se pelo alcance do desempenho esperado em cada série e parte da ação realizada pelo estudante na prática da aprendizagem.

O desempenho, segundo os estudiosos ${ }^{4,6,9-12}$, representa a ação desenvolvida pelo estudante por meio da mobilização dos recursos afetivos, cognitivos e psicomotores para a realização da tarefa a ele designada, segundo os critérios preestabelecidos e seus respectivos indicadores. Nessa perspectiva, o estudante é avaliado por meio de indicadores que são as pistas que evidenciam o alcance ou não da competência profissional.

A Famema, ao estruturar essa organização curricular, precisou empreender uma redefinição conceitual das relações no mundo do trabalho com todas as suas implicações subjetivas, de modo a institucionalizar as novas possibilidades da formação profissional. Nesta visão institucional, o currículo comprometido com o desenvolvimento da competência deve partir da análise do processo de trabalho para a construção de uma matriz referencial organizada pedagogicamente, em uma proposta organizativa corroborada pela literatura ${ }^{13}$

Partir do mundo do trabalho, à luz das DCN e do SUS, para organizar a proposta educativa, como a Famema o faz, tendo em vista os saberes necessários à formação médica, implica entender as complexidades inerentes aos âmbitos da educação e da gestão, e compreender as especificidades do trabalho das equipes e as dimensões sócio e ético-políticas que revelam a capacidade de refletir sobre a esfera do mundo do trabalho e o exercício da cidadania.

Para tanto, a Famema, considerando a construção do conhecimento com base no mundo do trabalho, organizou a proposta de ensino-aprendizagem em Unidade de Prática Profissional (UPP), que contempla os seguintes cenários: Laboratório de Prática Profissional (LPP), em que as atividades simuladas são realizadas em ambiente protegido, sendo o cenário real a Unidade de Saúde da Família (USF), que propicia a vivência da prática.

Esta compreensão insere a avaliação para o desenvolvimento de competência num contexto que a torna reflexiva, investigativa, contínua, participativa e negociada, devendo ser ancorada em uma relação professor-estudante democrática, dialogada e de reciprocidade.

Este entendimento do processo avaliativo deve se estruturar em um referencial de competência que envolve a mobilização de capacidades decisórias para a realização de ações específicas da prática. Sendo assim, o processo avaliativo expressa que o profissional deve ser capaz de realizar seu trabalho dentro dos padrões de qualidade referenciados pela sociedade ${ }^{9}$.

Tal concepção dialógica de competência concebe o desenvolvimento de recursos cognitivos, psicomotores e afetivos que, combinados, conformam distintas maneiras de realizar uma atividade. Essa abordagem, complexa e ampliada, constrói-se no diálogo entre a formação e o mundo do trabalho ${ }^{13}$

A prática da avaliação da aprendizagem deve contemplar o contexto de uso da competência, uma vez que a relação professor-estudante durante o processo de aprendizagem perpassa as dimensões sociais, éticas e políticas ${ }^{15}$.

Considerando toda essa complexidade, Perrenoud ${ }^{14}$ alerta que é mais fácil avaliar o conhecimento de um estudante do que a competência porque, para apreendê-la, deve-se observá-lo em tarefas complexas, o que exige tempo e abre caminho à contestação. Portanto, para avaliar a competência, a escola deverá utilizar, de modo cooperativo, a inventividade, situando o estudante na realidade, exigindo uma abordagem qualitativa e a observação de fatos e gestos, palavras e raciocínios, suas decisões e caminhos frente a um problema ${ }^{12}$. 
Hoffmann ${ }^{15}$ reforça que a avaliação deve ocorrer por meio de um elo interativo e dialógico entre professor e estudante e num processo de abertura e constante revisão, que se destina a conhecer para promover ações em benefício do educando. Ela ocorre fundamentalmente pela proximidade entre quem educa e quem é educado. Hoffmann ${ }^{16}$ assim descreve a ação transformadora da avaliação:

A avaliação é a reflexão transformada em ação. Ação essa que nos impulsiona a novas reflexões. Reflexão permanente do educador sobre sua realidade e acompanhamento, passo a passo, do educando, na sua trajetória de construção do conhecimento. Um processo interativo, através do qual educando e educadores aprendem sobre si mesmos e sobre a realidade escolar no ato próprio da avaliação. (p.18)

Desse modo, na proposta pedagógica da Famema, a avaliação da aprendizagem é compreendida como uma das atividades mais complexas porque guarda relação intrínseca com valores, condutas, perspectivas do mundo e ideologias. Nesse contexto, ela busca estruturar os critérios de avaliação de modo flexível, tendo em vista a compreensão do estudante e do professor sobre as pistas necessárias que servem de guia para verificar se o estudante atingiu o desempenho esperado. Essas pistas são os indicadores apresentados na Conferência de Consenso.

$\mathrm{Na}$ Conferência de Consenso, as discussões se nortearam pela busca da clarificação do indicador e da abrangência do desempenho, levando em conta o domínio de cada série do curso. A reelaboração dos indicadores resultou do entendimento dos participantes sobre sua função - servir ao monitoramento do processo - e de que a especificidade do indicador deverá estar contemplada e ancorada nos desempenhos esperados e nos instrumentos de avaliação de cada série.

Como resultado, os indicadores obtidos por consenso para avaliação da aprendizagem nas áreas de "organização e gestão do processo de trabalho em saúde" foram organizados em dois desempenhos, apresentados no Quadro 1.

\section{QuADRO 1}

Desempenho: organiza e elabora o trabalho em saúde junto à equipe

Indicadores da ação: identifica e analisa as diversas situações da gestão e do processo de trabalho em saúde

Caracteriza os níveis de atenção à saúde, identificando as relações de fluxo e de complexidade entre atenção primária, secundária e terciária em todos os cenários de ensino-aprendizagem.
Caracteriza a organização e a gestão do trabalho em saúde, pautado nos princípios do SUS, considerando os recursos humanos, financeiros e materiais/equipamentos.

Participa do processo de trabalho em saúde: discute os problemas e planos de intervenção, busca soluções em conjunto, estabelece cooperação com a equipe e se responsabiliza pelo acompanhamento das famílias.

Na Famema, assim como preconizado nas DCN, alguns saberes requeridos na formação médica dizem respeito a gerenciamento, administração e liderança, que implicam também habilidade de comunicação, tomada de decisão e organização do trabalho em equipe multiprofissional e, ainda, capacidade de tomar as melhores iniciativas. Esse conhecimento gerencial pode favorecer o processo de produção em saúde, tanto para o trabalho dos profissionais, quanto para a população atendida, visto que extrapola o corporativismo para um projeto coletivo, privilegiando o usuário como centro do processo gerencial e de atenção ${ }^{1,17}$.

Segundo Carvalho e Cunha ${ }^{18}$, o modelo de atenção permite perceber que diferentes composições de recursos produzem resultados diversificados em saúde, ou seja, em duas cidades diferentes com a mesma quantidade de profissionais, recursos financeiros e materiais podem produzir resultados diversos, dependendo da forma como estes se articulam. Esses autores corroboram a ideia de que os três indicadores acima estabelecidos são coerentes para se demonstrar o alcance dos estudantes em relação ao critério definido.

No que se refere à ação de elaborar e executar o plano de intervenção para organização do processo de trabalho em saúde, foram apontados os indicadores apresentados no Quadro 2.

\section{QUADRO 2}

Desempenho: organiza e elabora o trabalho em saúde junto à equipe

Indicadores da ação: elabora e executa o plano de intervenção

Elabora o plano de trabalho com a equipe, estabelecendo metas, pautando-se em ações de vigilância à saúde: promoção à saúde, prevenção de doença, recuperação e reabilitação.

Executa o plano elaborado.

Realiza a avaliação formativa e somativa, retroalimentando o plano de intervenção.

Nesse contexto, os três indicadores obtidos por consenso para avaliação da aprendizagem no âmbito da gestão são 
coerentes para demonstrar o alcance dos estudantes em relação ao critério definido. Segundo as DCN, o processo de ensino-aprendizagem deve permitir ao estudante conhecer e vivenciar situações variadas de vida, da organização da prática e do trabalho em equipe multiprofissional, a fim de contribuir para que ele aprenda a lidar com problemas reais e assuma responsabilidades crescentes como agente prestador de cuidados e atenção, compatíveis com seu grau de autonomia ${ }^{1}$.

Campos e colaboradores ${ }^{19}$ demonstram que a especialização e a multiplicação de profissionais que intervêm de modo fragmentado em cada caso são fatores que dificultam a construção de vínculo, a responsabilidade sanitária e a clínica compartilhada. Nesse sentido, há importante consenso para que ocorra a reformulação tanto do processo de trabalho e de gestão em saúde como da relação horizontal no tempo, estabelecendo-se uma clara definição de responsabilidades entre trabalhadores e usuários. Essa problemática inerente ao SUS, e apresentada pelos autores, indica que as mudanças na educação médica podem colaborar para reverter este quadro.

No que diz respeito à ação de desenvolver o trabalho em grupo, a discussão na Conferência de Consenso destacou os indicadores apresentados no Quadro 3.

\section{QuADRO 3}

Desempenho: identifica necessidades coletivas de saúde

\section{Indicadores da ação: desenvolve o trabalho em grupo}

Estabelece comunicação efetiva, respeita os princípios e valores dos membros do grupo e desempenha suas tarefas com responsabilidade.

Realiza a autoavaliação e a de interpares.

As $\mathrm{DCN}^{1}$ indicam que se deve desenvolver a capacidade de aprender a aprender, a responsabilidade e o compromisso com a formação do médico e a das futuras gerações de profissionais, assim como salientam a importância da ética nas relações humanas e da comunicação verbal, não verbal, interpessoal e, ainda, o domínio das tecnologias de informação e comunicação.

Contribuindo com este resultado, na literatura, Masetto ${ }^{20}$ propõe que a relação entre professor e estudantes parta da consideração de que ambos são capazes de assumir um processo de aprendizagem e que o estudante está apto a trabalhar em parceria com o professor e com os outros colegas para aprender. Prioriza a participação efetiva em todas as atividades programadas, com colaboração fundamentada, visando desenvolver aprendizagem significativa por meio da defini- ção clara de objetivos e metas, bem como do uso de recursos adequados e eficientes. Para que isso aconteça, é necessário criar um sistema de avaliação contínuo e pactuado com todos os atores envolvidos no processo de ensino-aprendizagem.

Para o desempenho de identificar necessidades coletivas de saúde, os indicadores obtidos por consenso para a ação de coletar dados epidemiológicos e realizar o diagnóstico são os apresentados no Quadro 4.

\section{QUADRO 4}

Desempenho: identifica necessidades coletivas de saúde

Indicadores da ação: coleta dados epidemiológicos e realiza diagnóstico situacional

Identifica e utiliza fontes de informação primária e secundária e participa da análise dos dados em conjunto com a equipe de saúde, à luz da epidemiologia descritiva e social.

Identifica e utiliza os indicadores econômicos, sociais e de saúde para o diagnóstico e planejamento de ações nos diversos cenários de atenção.

Identifica os equipamentos sociais para o planejamento das intervenções nos diversos cenários em diferentes níveis de atenção.

Identifica os fatores relacionados ao ambiente, à saúde ocupacional e às políticas de saúde para a compreensão do processo saúde-

doença e utiliza-os em todos os cenários.

Realiza diagnóstico de saúde da população e planeja intervenção, considerando as condições do serviço e a realidade socioeconômico-cultural da área de abrangência, correlacionandoas com os problemas das pessoas e das famílias acompanhadas, segundo o grau de autonomia do estudante e domínio para cada série.

Elabora um relatório do diagnóstico de saúde de acordo com as normas da ABNT.

Reconhece fatores que facilitam ou limitam o trabalho profissional na transformação dos problemas de saúde.

Realiza diagnóstico epidemiológico situacional na perspectiva das necessidades coletivas de saúde, considerando o grau de autonomia do estudante e domínio para cada série.

Prioriza os problemas levantados.

Elabora um plano de intervenção junto com a equipe, de acordo com as pessoas envolvidas, considerando o grau de autonomia do estudante e o domínio para cada série.

Executa o plano de intervenção e avalia o processo, retroalimentando o plano.

A qualidade da formação deve estar envolvida com o papel social e político do trabalho em saúde. A formação implica a condição de refazer permanentemente as relações profissionais com os usuários de modo responsável e comprometido. 
Esse perfil se alinha ao que preconizam o SUS e as DCN para a formação do médico em nosso país.

Carvalho e Ceccim ${ }^{21}$ sugerem que a formação em saúde coletiva deve trabalhar pela implicação dos estudantes em determinadas práticas: de cuidado individual e coletivo; de afirmação da vida, sob todas as suas formas inventivas e mais criativas de saúde; de responsabilidade com as pessoas e coletividades por melhor saúde individual e coletiva; de desenvolvimento e realização de um sistema de saúde com capacidade de proteção à vida e à saúde; e, ainda, práticas de participação e solidariedade que incluam projetos de democracia, cidadania e direitos sociais. Além de dominar os processos lógicos de construção dos saberes profissionais, os meios, técnicas e métodos de produção de conhecimento científico que fundamentam e orientam cada atuação profissional, o estudante deve saber mobilizar, articuladamente, esses conhecimentos científicos, transformando-os em atividade social e política libertadora.

Os autores também alertam que o profissional precisa saber avaliar criticamente sua própria atuação e o contexto em que atua, para interagir ativamente pela cooperação entre os colegas de trabalho, constituindo coletivos de produção da saúde mediante o sentir o outro na relação com os usuários dos serviços em que atua ou sob a mediação com as instâncias da sociedade que participam do controle social em saúde.

\section{CONCLUSÃO}

O desenvolvimento desta pesquisa levou à elaboração de indicadores para avaliação critério-referenciada do desempenho do estudante no processo de ensino-aprendizagem nas áreas de gestão e de saúde coletiva do curso de Medicina da Famema. Essa construção possibilita ampliar e aprofundar a avaliação nessas áreas específicas da formação médica, considerando o cenário de aprendizagem.

O processo descrito para estruturar o caminho da elaboração de indicadores, com a participação de diferentes olhares, pode fornecer novos subsídios para a organização do processo avaliativo na formação profissional.

Ficou também evidenciado que a abordagem qualitativa, por meio da Conferência de Consenso, mostrou-se efetiva para o entendimento dos indicadores necessários ao processo avaliativo desenvolvido.

Acreditamos que o conhecimento desses indicadores pelos gestores dos serviços de saúde que recebem estes estudantes permitirá maior compreensão da proposta do curso de Medicina e das expectativas dos estudantes, de forma que tais indicadores possam atuar como facilitadores do processo de aprendizagem, pois isso traria maior clareza quanto aos papéis dos estudantes e tornaria mais coesa sua atuação. Tal sintonia possibilitará a formação integral, visando à competência profissional por meio da efetiva articulação entre as séries, e a construção do conhecimento com base no mundo do trabalho.

Dessa forma, o estudo possibilita caminhar no sentido da implementação das alterações curriculares, com ênfase na avaliação da aprendizagem que se compromete com um processo formativo preocupado com a melhoria da qualidade da formação médica e da assistência em saúde em nosso país.

\section{REFERÊNCIAS}

1. Brasil. Ministério da Educação. Conselho Nacional de Educação. Câmara de Educação Superior. Parecer CNE/CES no. 1133 de 7 agosto de 2001. Institui as diretrizes curriculares nacionais dos cursos de graduação em enfermagem, medicina e nutrição. Diário Oficial da União. Brasília, 3 out. 2001. [acesso em 12 fev. 2009]. Disponível em: <http:/ / www.mec. gov.br/Sesu/diretriz.shtm\#legislação>.

2. Faculdade de Medicina de Marília. Manual de avaliação do estudante: Cursos de Medicina e Enfermagem. Marília: Famema; 2012.

3. Ceccim RB, Pinto LF. A formação e especialização de profissionais de saúde e a necessidade política de enfrentar as desigualdades sociais e regionais. Rev Bras Educ Med. 2007;31(3):266-77.

4. Lima VV. Competence: different approaches and implications in the training of healthcare professionals. Interface Comun Saúde Educ. 2005;9(17)369-79.

5. Gomes R, Francisco AM, Tonhom SFR, Costa MCG, Hamamoto CG, Pinheiro OL, et al. A formação médica ancorada na aprendizagem baseada em problema: uma avaliação qualitativa. Interface Comun Saúde Educ. 2009;13(28):7183.

6. Minayo MCS. Construção de indicadores qualitativos para avaliação de mudanças. Rev Bras Educ Med. 2009;33(1 Supl. 1):S 83-91.

7. Almeida MJ, Campos JJB, Turini B, Nicoletto SCS, Pereira LA, Rezende LR. et al. Implantação das Diretrizes Curriculares Nacionais na Graduação em Medicina no Paraná. Rev Bras Educ Med. 2007;31(2):156-65.

8. Souza LEPF, Vieira-da-Silva LM, Hartz ZMA. Conferência de consenso sobre a imagem-objetivo da descentralização da atenção à saúde no Brasil. In: Hartz ZMA, Vieira-da-Silva LM (orgs.). Avaliação em saúde: dos modelos teóricos à prática na avaliação de programas e sistemas de saúde. Rio de Janeiro: Ed. Fiocruz; Salvador: EDUFBA; 2005. p. 65-102. 
9. Hager P, Gonczi A. What is competence? Med Teach (London). 1996;18(1):15-8.

10. Ramos MN. A Pedagogia das competências: autonomia ou adaptação? 3ª.ed. São Paulo: Cortez; 2006.

11. Depresbiteres L. Avaliação da aprendizagem: casos comentados. Pinhais: Melo; 2011.

12. Guimarães APC, Taipeiro EF, Higa EFR. Avaliação no âmbito da graduação: contextualização histórica e desafios da contemporaneidade. In: Moraes MAA, Tonhom SFR, Hafner MLMB, Gomes R (orgs). Avaliação nos Cursos de Medicina e Enfermagem: perspectivas e desafios. Curitiba: CRV; 2012. p.75-105.

13. Ramos MN. A educação profissional pela pedagogia das competências e a superfície dos documentos oficiais. Educ. Soc.2002; 23(80) 401-22.

14. Perrenoud P. Construir as competências desde a escola. Porto Alegre: Artes Médicas Sul; 1999.

15. Hofmann J M L. Avaliar para promover: as setas do caminho. $7^{a}$ ed. Porto Alegre: Mediação; 2001.

16. Hofmann J M L. Avaliação mito e desafio: uma perspectiva construtivista. 18aed. Porto Alegre: Mediação; 1995.

17. Campos GWS. Equipes de referência e apoio especializado matricial: um ensaio sobre a reorganização do trabalho em saúde. Cienc Saúde Coletiva. 1999;4(2):393-403.

18. Carvalho SR, Cunha GT, A gestão da atenção na saúde: elementos para se pensar a mudança da organização na saúde. In: Campos GWS, Minayo MCS, Akerman M, Drumond Junior M, Carvalho YM. Tratado de Saúde Coletiva. $2^{\underline{a}}$ reimpr. São Paulo: Hucitec; Rio de Janeiro: Ed. Fiocruz; 2007.
19. Campos G W S, Minayo M C S, Akerman M, Drumond Júnior M, Carvalho Y M (orgs.). Tratado de Saúde Coletiva. São Paulo: Hucitec; Rio de Janeiro: Fiocruz; 2006.

20. Masetto MT Competência pedagógica do professor universitário. São Paulo: Summus; 2003.

21. Carvalho YM, Cecim RB. Formação e educação em saúde: aprendizados com a saúde coletiva. In: Campos G W S, Minayo M C S, Akerman M, Drumond Júnior M, Carvalho Y M (orgs.). Tratado de saúde coletiva. São Paulo: Hucitec; Rio de Janeiro: Fiocruz; 2006. p137-70.

\section{CONTRIBUIÇÃO DOS AUTORES}

Todos participaram na elaboração do projeto e na coleta de dados. A elaboração do artigo foi prioritariamente conduzida por Elza de Fátima R. Higa e Maria de Lourdes M. B. Hafner.

\section{CONFLITO DE INTERESSES}

Declarou não haver.

\section{ENDEREÇO PARA CORRESPONDÊNCIA}

Elza de Fátima Ribeiro Higa

Rua Davino Alves de Souza, 112

Maria Isabel - Marília

CEP: 17516-230 SP

E-mail: hirifael@gmail.com 